\title{
Numerical Analysis of Fractional Order Epidemic Model of Childhood Diseases
}

\author{
Fazal Haq, ${ }^{1}$ Muhammad Shahzad, ${ }^{1}$ Shakoor Muhammad, \\ Hafiz Abdul Wahab, ${ }^{1}$ and Ghaus ur Rahman ${ }^{3}$ \\ ${ }^{1}$ Department of Mathematics, Hazara University, Mansehra, Khyber Pakhtunkhwa, Pakistan \\ ${ }^{2}$ Department of Mathematics, Abdul Wali Khan University, Mardan, Pakistan \\ ${ }^{3}$ Department of Mathematics and Statistics, University of Swat, Khyber Pakhtunkhwa, Pakistan
}

Correspondence should be addressed to Fazal Haq; fazalhaqphd@gmail.com

Received 26 July 2017; Revised 4 October 2017; Accepted 5 November 2017; Published 3 December 2017

Academic Editor: Stefania Tomasiello

Copyright (C) 2017 Fazal Haq et al. This is an open access article distributed under the Creative Commons Attribution License, which permits unrestricted use, distribution, and reproduction in any medium, provided the original work is properly cited.

The fractional order Susceptible-Infected-Recovered (SIR) epidemic model of childhood disease is considered. Laplace-Adomian Decomposition Method is used to compute an approximate solution of the system of nonlinear fractional differential equations. We obtain the solutions of fractional differential equations in the form of infinite series. The series solution of the proposed model converges rapidly to its exact value. The obtained results are compared with the classical case.

\section{Introduction}

Childhood diseases are most serious infectious diseases. Measles, poliomyelitis, and rubella are famous among them $[1,2]$. Measles is a highly infectious disease, caused by respiratory infection by a Morbillivirus. These diseases normally affect the children, because child population is more prone to the disease as compared to the adults [1]. Therefore, the population can be divided into two major classes: premature and mature populations. Premature population takes a constant time to become mature, which is known as maturation delay. In disease dynamics, a disease cannot spread instantaneously but rather it will take some time in the body which is called latent period for the particular disease. For the control of childhood disease vaccination is a significant strategy being used all over the world. A universal effort to extend vaccination range to all children began in 1974, when the World Health Organization (WHO) founded the Expanded Program on Immunization [3]. Mathematical model plays an important role to comprehend the process of transmission of a disease and provides different techniques to control its propagation. Many mathematicians investigated childhood disease; for instance, Singh et al. [2] studied about vaccination of the childhood diseases. In addition, the authors presented numerical solution of the childhood disease model. Makinde [4] presented a Susceptible-Infected-Recovered model

$$
\begin{aligned}
& \frac{d s}{d t}=(1-p) \pi-\beta \frac{s i}{n}-\pi s, \\
& \frac{d i}{d t}=\beta \frac{s i}{n}-(\gamma+\pi) i, \\
& \frac{d r}{d t}=p \pi+\gamma i-\pi r .
\end{aligned}
$$

The authors [1] rearranged model (1) using the relations $s / n=$ $S, i / n=I$, and $r / n=R$ and obtained a new (SIR) model in the following form:

$$
\begin{aligned}
& \frac{d S}{d t}=(1-p) \pi-\beta S I-\pi S, \\
& \frac{d I}{d t}=\beta S I-(\gamma+\pi) I, \\
& \frac{d R}{d t}=p \pi+\gamma I-\pi R .
\end{aligned}
$$

The description of the preceding model is given below. 
The model shows that vaccination is 100 percent efficient and the natural death rate $\mu$ is unequal. Therefore the total population size $N$ is not constant. The birth rate is represented by $\pi$ while the rate of mortality of the childhood disease is very low. The parameter, $p$, represents a fraction of vaccinated population at birth, where $0<p<1$, considering that the rest of population is susceptible. A susceptible individual suffers from the disease through a contact with infected individuals at rate $\beta$. Infected individuals recover at a rate $\gamma$. There are many applications of fractional calculus $[5,6]$. Mathematicians and researchers used fractional calculus to model real world problems. Fractional calculus has greater degree of freedom; therefore it helps to solve nonlinear problems $[7,8]$. Also with the help of fractional derivative interdisciplinary applications can be studied. The nonlinear oscillation of earthquake can be modeled with the help of fractional derivative.

In 1980, Laplace-Adomian Decomposition Method (LADM) was introduced by Adomian, which is an effective method for finding the numerical and explicit solution of a system of differential equations representing physical problems. This method works efficiently for solving several kinds of differential equations. It includes nonlinear boundary value/initial value problems. Moreover, we can use it for the partial differential equations; in addition, it can be used to solve a system of stochastic differential equations also. In this method, no perturbation or linearization is required. The advantage of LADM is that it needs no extra memory and does not require any additional parameters, which wastes time and memory.

Studying the literature review of childhood disease model it has been found that Arafa et al. [1] presented the fractional order model for the childhood disease and provided numerical solution by using homotopy analysis method. The reason behind the use of fractional order differential equations (FDEs) is that FDEs are naturally related to systems that involve memory, which can be found in many biological systems. Also, they show the realistic behavior of infection of disease but at a slower rate. Motivated by the applications of fractional calculus and LADM we explore numerical solution of childhood disease model. The Caputo derivative, which is a modification of the Riemann-Liouville definition $[9,10]$, is considered as a differential operator in our model.

We now gather some well-known definitions and results from the literature, which we will use throughout this paper. For more details, we refer the reader to $[1,7-12]$.

Definition 1. The Caputo fractional order derivative of a function $y$ on the interval $[0, T]$ is defined by

$$
{ }^{c} D_{0+}^{\alpha} y(t)=\frac{1}{\Gamma(n-\alpha)} \int_{0}^{t}(t-s)^{n-\alpha-1} y^{(n)}(s) d s,
$$

where $n=[\alpha]+1$ and $[\alpha]$ represents the integer part of $\alpha$.

Definition 2. We recall the definition of Laplace transform of Caputo derivative as

$$
\begin{array}{r}
\mathscr{L}\left\{{ }^{c} D^{\alpha} y(t)\right\}=s^{\alpha} y(s)-\sum_{k=0}^{n-1} s^{\alpha-k-1} y^{(k)}(0), \\
n-1<\alpha<n, n \in N .
\end{array}
$$

\section{The Laplace-Adomian Decomposition Method}

Using the Caputo fractional derivative system (2) gets the following form:

$$
\begin{aligned}
& { }^{c} D^{\alpha} S(t)=(1-p) \pi-\beta S I-\pi S, \\
& { }^{c} D^{\alpha} I(t)=\beta S I-(\gamma+\pi) I, \\
& { }^{c} D^{\alpha} R(t)=p \pi+\gamma I-\pi R,
\end{aligned}
$$

where $\alpha \in(0,1]$ while $\pi, \gamma, p$, and $\beta$ are positive parameters and the given initial conditions are $S(0)=N_{1}, I(0)=N_{2}$, and $R(0)=N_{3}$. To solve system (5), we use Laplace-Adomian Decomposition Method (LADM) [5, 13]. Moreover, the obtained solution will be compared with the integer order derivative case. Furthermore, we use Laplace transformation to convert the system of differential equations into a system of algebraic equations. Then, the algebraic equations are used to obtain the required solution in form of series. We will discuss the procedure for solving model (5) with given initial conditions. Applying Laplace transform on both sides of model (5), we obtain the following system:

$$
\begin{aligned}
& \mathscr{L}\left\{{ }^{c} D^{\alpha} S(t)\right\}=\mathscr{L}\{(1-p) \pi-\beta S I-\pi S\}, \\
& \mathscr{L}\left\{{ }^{c} D^{\alpha} I(t)\right\}=\mathscr{L}\{\beta S I-(\gamma+\pi) I\}, \\
& \mathscr{L}\left\{{ }^{c} D^{\alpha} R(t)\right\}=\mathscr{L}\{p \pi+\gamma I-\pi R\},
\end{aligned}
$$

or

$$
\begin{aligned}
& s^{\alpha} \mathscr{L}\{S(t)\}-s^{\alpha-1} S(0)=\mathscr{L}\{(1-p) \pi-\beta S I-\pi S\}, \\
& s^{\alpha} \mathscr{L}\{I(t)\}-s^{\alpha-1} I(0)=\mathscr{L}\{\beta S I-(\gamma+\pi) I\}, \\
& s^{\alpha} \mathscr{L}\{R(t)\}-s^{\alpha-1} R(0)=\mathscr{L}\{p \pi+\gamma I-\pi R\} .
\end{aligned}
$$

Using the initial conditions (7), we obtain the form

$$
\begin{aligned}
& \mathscr{L}\{S(t)\}=\frac{S_{0}}{s}+\left[\frac{1}{s^{\alpha}} \mathscr{L}\{(1-p) \pi-\beta S I-\pi S\}\right], \\
& \mathscr{L}\{I(t)\}=\frac{I_{0}}{s}+\left[\frac{1}{s^{\alpha}} \mathscr{L}\{\beta S I-(\gamma+\pi) I\}\right], \\
& \mathscr{L}\{R(t)\}=\frac{R_{0}}{s}+\left[\frac{1}{s^{\alpha}} \mathscr{L}\{p \pi+\gamma I-\pi R\}\right] .
\end{aligned}
$$

Assume that the solutions $S(t), I(t)$, and $R(t)$ in the form of infinite series are given by 


$$
\begin{aligned}
& S(t)=\sum_{k=0}^{\infty} S_{k}(t), \\
& I(t)=\sum_{k=0}^{\infty} I_{k}(t), \\
& R(t)=\sum_{k=0}^{\infty} R_{k}(t),
\end{aligned}
$$

while the nonlinear term $S(t) I(t)$ is decomposed as follows:

$$
S(t) I(t)=\sum_{k=0}^{\infty} A_{k}(t)
$$

where each $A_{k}$ is the Adomian polynomials defined as

$$
A_{k}=\left.\frac{1}{\Gamma(k+1)} \frac{d^{k}}{d \lambda^{k}}\left[\sum_{j=0}^{k} \lambda^{j} S_{j}(t) \sum_{j=0}^{k} \lambda^{j} I_{j}(t)\right]\right|_{\lambda=0} .
$$

The first three polynomials are given by

$$
\begin{aligned}
& A_{0}=S_{0}(t) I_{0}(t), \\
& A_{1}=S_{0}(t) I_{1}(t)+S_{1}(t) I_{0}(t), \\
& A_{2}=2 S_{0}(t) I_{2}(t)+2 S_{1}(t) I_{1}(t)+2 S_{2}(t) I_{0}(t) .
\end{aligned}
$$

Substituting (9) and (10) into (8) results in

$$
\begin{aligned}
& \mathscr{L}\left\{\sum_{k=0}^{\infty} S_{k}(t)\right\} \\
& =\frac{S_{0}}{s} \\
& +\left[\frac{1}{s^{\alpha}} \mathscr{L}\left\{(1-p) \pi-\beta \sum_{k=0}^{\infty} A_{k}(t)-\pi \sum_{k=0}^{\infty} S_{k}(t)\right\}\right], \\
& \mathscr{L}\left\{\sum_{k=0}^{\infty} I_{k}(t)\right\} \\
& =\frac{I_{0}}{s}+\left[\frac{1}{s^{\alpha}} \mathscr{L}\left\{\beta \sum_{k=0}^{\infty} A_{k}(t)-(\gamma+\pi) \sum_{k=0}^{\infty} I_{k}(t)\right\}\right], \\
& \mathscr{L}\left\{\sum_{k=0}^{\infty} R_{k}(t)\right\} \\
& =\frac{R_{0}}{s}+\left[\frac{1}{s^{\alpha}} \mathscr{L}\left\{p \pi+\gamma \sum_{k=0}^{\infty} I_{k}(t)-\pi \sum_{k=0}^{\infty} R_{k}(t)\right\}\right] .
\end{aligned}
$$

Matching the two sides of (13) yields the following iterative algorithm:

$$
\begin{gathered}
\mathscr{L}\left(S_{0}\right)=\frac{N_{1}}{s}, \\
\mathscr{L}\left(S_{1}\right)=\frac{(1-p) \pi}{s^{\alpha}}-\frac{\beta}{s^{\alpha}} \mathscr{L}\left\{A_{0}\right\}-\frac{\pi}{s^{\alpha}} \mathscr{L}\left\{S_{0}\right\}, \\
\mathscr{L}\left(S_{2}\right)=\frac{(1-p) \pi}{s^{\alpha}}-\frac{\beta}{s^{\alpha}} \mathscr{L}\left\{A_{1}\right\}-\frac{\pi}{s^{\alpha}} \mathscr{L}\left\{S_{1}\right\},
\end{gathered}
$$

$$
\mathscr{L}\left(S_{k+1}\right)=\frac{(1-p) \pi}{s^{\alpha}}-\frac{\beta}{s^{\alpha}} \mathscr{L}\left\{A_{k}\right\}-\frac{\pi}{s^{\alpha}} \mathscr{L}\left\{S_{k}\right\},
$$

$k \geq 1$,

$$
\begin{gathered}
\mathscr{L}\left(I_{0}\right)=\frac{N_{2}}{s}, \\
\mathscr{L}\left(I_{1}\right)=\frac{\beta}{s^{\alpha}} \mathscr{L}\left\{A_{0}\right\}-\frac{\gamma+\pi}{s^{\alpha}} \mathscr{L}\left\{I_{0}\right\}, \\
\mathscr{L}\left(I_{2}\right)=\frac{\beta}{s^{\alpha}} \mathscr{L}\left\{A_{1}\right\}-\frac{\gamma+\pi}{s^{\alpha}} \mathscr{L}\left\{I_{1}\right\},
\end{gathered}
$$

$$
\begin{gathered}
\mathscr{L}\left(I_{k+1}\right)=\frac{\beta}{s^{\alpha}} \mathscr{L}\left\{A_{k}\right\}-\frac{\gamma+\pi}{s^{\alpha}} \mathscr{L}\left\{I_{k}\right\}, \quad k \geq 1, \\
\mathscr{L}\left(R_{0}\right)=\frac{N_{3}}{s},
\end{gathered}
$$

$$
\begin{gathered}
\mathscr{L}\left(R_{1}\right)=\frac{p \pi}{s^{\alpha}}+\frac{\gamma}{s^{\alpha}} \mathscr{L}\left\{I_{0}\right\}-\frac{\pi}{s^{\alpha}} \mathscr{L}\left\{R_{0}\right\}, \\
\vdots \\
\mathscr{L}\left(R_{k+1}\right)=\frac{p \pi}{s^{\alpha}}+\frac{\gamma}{s^{\alpha}} \mathscr{L}\left\{I_{k}\right\}-\frac{\pi}{s^{\alpha}} \mathscr{L}\left\{R_{k}\right\}, \quad k \geq 1 .
\end{gathered}
$$

Taking Laplace inverse of (14) and considering first three terms, we get

$$
\begin{aligned}
S_{0} & =N_{1}, \\
S_{1} & =(1-p) \pi \frac{t^{\alpha}}{\Gamma(\alpha+1)}-\beta\left(N_{1} N_{2}+N_{1} \pi\right) \frac{t^{\alpha}}{\Gamma\left(t^{\alpha}+1\right)} \\
& -(1-p) \pi^{2} \frac{t^{2 \alpha}}{\Gamma(2 \alpha+1)}, \\
S_{2} & =(1-p) \pi \frac{t^{\alpha}}{\Gamma(\alpha+1)}-\beta^{2}\left(N_{1}^{2} N_{2}\right. \\
& \left.+\beta(\gamma+\pi) N_{1} N_{2}\right) \frac{t^{2} \alpha}{\Gamma\left(t^{2} \alpha+1\right)}-\beta\left(N_{1} N_{2}+N_{1} \pi\right)
\end{aligned}
$$




$$
\begin{aligned}
& \cdot\left(\beta N_{2} \pi\right) \frac{t^{2 \alpha}}{\Gamma(\alpha+1)}-(1-p) \pi^{2}\left(\beta N_{2}+\pi\right) \\
\cdot & \frac{t^{3 \alpha}}{\Gamma(3 \alpha+1)}, \\
I_{0}= & N_{2}, \\
I_{1}= & \beta N_{1} N_{2} \frac{t^{\alpha}}{\Gamma\left(t^{\alpha}+1\right)}-(\gamma+\pi) N_{2} \frac{t^{\alpha}}{\Gamma\left(t^{\alpha}+1\right)}, \\
I_{2}= & \beta N_{1} N_{2}\left(\beta N_{1} N_{2}-(\gamma+\pi)-(\gamma+\pi) \beta N_{1} N_{2}\right. \\
& \left.-(\gamma+\pi) N_{2}\right) \frac{t^{2 \alpha}}{\Gamma(2 \alpha+1)}-\beta N_{2}\left(\beta N_{1} N_{2}+\pi N_{1}\right) \\
& \cdot \frac{t^{2 \alpha}}{\Gamma(2 \alpha+1)}-\left((1-p) \pi^{2}-\left(\beta N_{2}+\pi\right)\right) \\
& \cdot \frac{t^{3 \alpha}}{\Gamma(3 \alpha+1)}, \\
R_{0} & =N_{3}, \\
R_{1} & =\left(p \pi-\gamma N_{2}-\gamma N_{3}\right) \frac{t^{\alpha}}{\Gamma(\alpha+1)}-p \pi \frac{t^{2 \alpha}}{\Gamma(2 \alpha+1)}, \\
R_{2}= & p \pi \frac{t^{\alpha}}{\Gamma(\alpha+1)}+\gamma\left(\beta N_{1} N_{2}-(\gamma+\pi)\right. \\
+ & \left.\left(\pi \gamma N_{2}-\pi^{2} N_{3}\right)\right) \frac{t^{2 \alpha}}{\Gamma(2 \alpha+1)}-p \pi^{2} \frac{t^{3 \alpha}}{\Gamma(3 \alpha+1)} .
\end{aligned}
$$

\section{Numerical Results and Discussion}

Using $N_{1}=1, N_{2}=0.5, N_{3}=0, \mu=0.4, \beta=0.8, \gamma=0.03$, $p=0.9$, and $\pi=0.4$, the LADM provides us with an approximate solution in the form of infinite series. Thus we calculate the first four terms of (5) to obtain

$$
\begin{aligned}
S(t)= & +0.4400 \frac{t^{\alpha}}{\Gamma(\alpha+1)}-0.0868 \frac{t^{2 \alpha}}{\Gamma(2 \alpha+1)} \\
& +0.0490 \frac{t^{3 \alpha}}{\Gamma(3 \alpha+1)}, \\
I(t)= & 0.2+0.2340 \frac{t^{\alpha}}{\Gamma(\alpha+1)}+0.0923 \frac{t^{2 \alpha}}{\Gamma(2 \alpha+1)} \\
& +0.0080 \frac{t^{3 \alpha}}{\Gamma(3 \alpha+1)}, \\
R(t)= & 1.0800 \frac{t^{\alpha}}{\Gamma(\alpha+1)}-0.3240 \frac{t^{2 \alpha}}{\Gamma(2 \alpha+1)} \\
& +0.0336 \frac{t^{3 \alpha}}{\Gamma(3 \alpha+1)} .
\end{aligned}
$$

For $\alpha=1,(16)$ attains the form

$$
\begin{aligned}
S(t)= & 1+0.4400 t-0.1278000 t^{2}+0.0081666668 t^{3}, \\
I(t)= & 0.2+0.2340 t+0.0465000 t^{2} \\
& +0.0003333668 t^{3}, \\
R(t)= & 1.0800 t-0.324000 t^{2}+0.0366000 t^{3} .
\end{aligned}
$$

Similarly, we get the following system for $\alpha=0.95$ :

$$
\begin{aligned}
S(t)= & +0.44903 t^{0.95}-0.1398742 t^{1.90} \\
& +0.0098280395 t^{2.85} \\
I(t)= & 0.2+0.23880453 t^{0.95}+0.050899322 t^{1.90} \\
& +0.000449848 t^{2.85} \\
R(t)= & 1.1021503 t^{0.95}-0.35461087 t^{1.90} \\
& +0.0400435362 t^{2.85}
\end{aligned}
$$

Now, for $\alpha=0.85$, one can obtain the following system:

$$
\begin{aligned}
S(t)= & +0.4653075 t^{0.85}-0.16547042 t^{1.70} \\
& +0.01394913 t^{2.55} \\
I(t)= & 0.2+0.2474402 t^{0.85}+0.06020641 t^{1.70} \\
& +0.00078925 t^{2.55} \\
R(t)= & 1.14211848 t^{0.85}-0.41950277 t^{1.70} \\
& +0.05738273 t^{2.55}
\end{aligned}
$$

Similarly, the solution after three terms for $\alpha=0.75$ is calculated as follows:

$$
\begin{aligned}
S(t)= & 1+0.44787487 t^{0.75}-0.1922758 t^{1.50} \\
& +0.0122128708 t^{2.55} \\
I(t)= & 0.2+0.254607 t^{0.75}+0.0699959508 t^{1.50} \\
& +0.00133334 t^{2.55} \\
R(t)= & 1.1751104 t^{0.75}-0.4874598003 t^{1.50} \\
& +0.790818664 t^{2.55}
\end{aligned}
$$

From the graphical results it is clear that the result obtained by using LADM is very efficient. It also shows that the presented method can predict the behavior of the variables accurately for the region under consideration. It is also clear that the efficiency of this method can be dramatically increased by increasing the terms. Fractional order derivative provides a greater degree of freedom as compared to integer order derivative. The dynamics of various compartments have been shown in Figure 1, Figure 2, and Figure 3, respectively. 


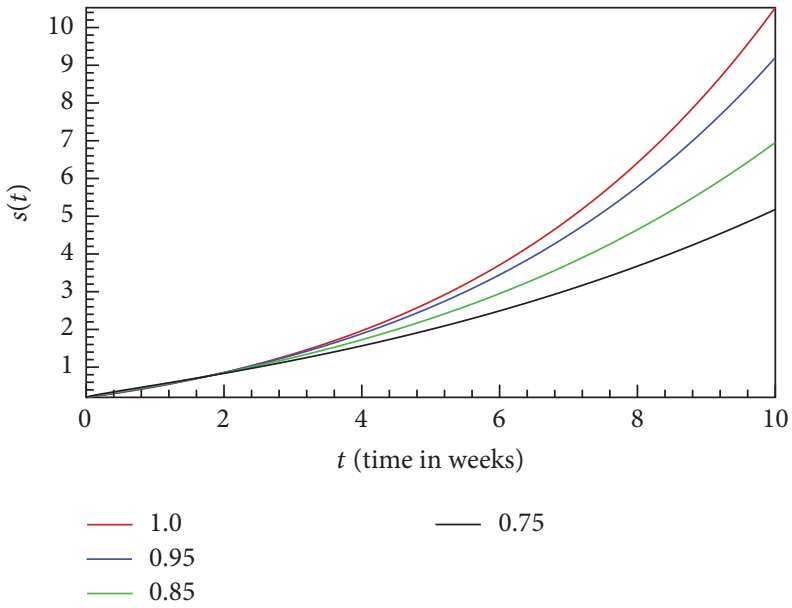

FIGURE 1: Plot of approximate solutions of susceptible class $(s)$ corresponding to different fractional values of $\alpha_{k}$ for $k=1,2,3$.

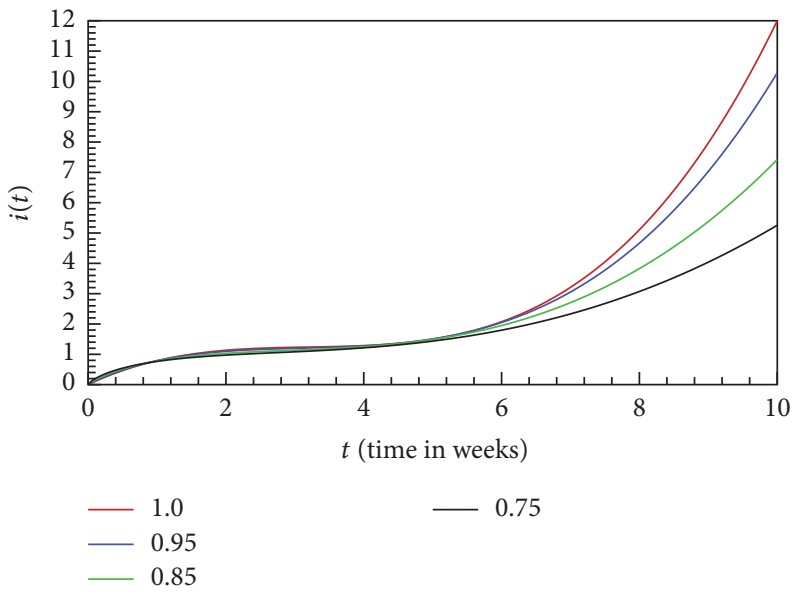

FIGURE 2: Plot of approximate solutions of infected class (i) corresponding to different fractional values of $\alpha_{k}$ for $k=1,2,3$.

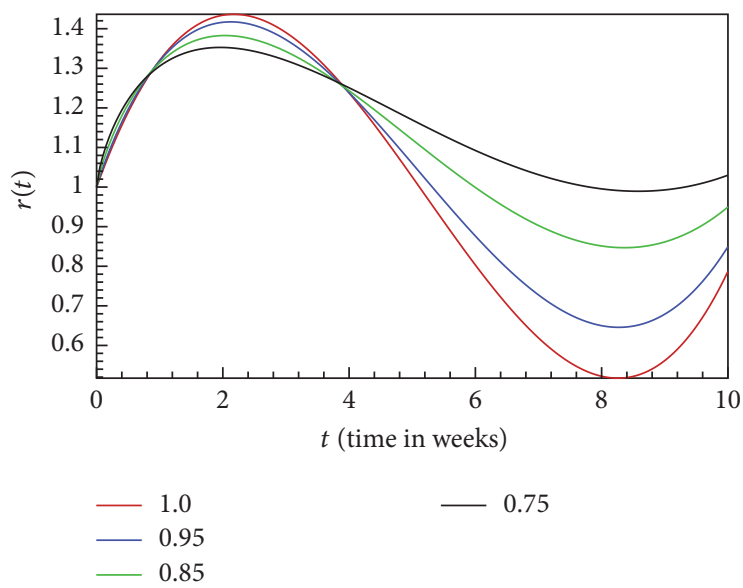

FIGURE 3: Plot of approximate solutions of removed class $(r)$ corresponding to different fractional values of $\alpha_{k}$ for $k=1,2,3$. 
TABLE 1: Numerical solution of the proposed model using LADM at classical order $\alpha=1$.

\begin{tabular}{lcrr}
\hline Time (week) & $S(t)$ & $I(t)$ & $R(t)$ \\
\hline$t=0$ & 1.00000 & 0.20000 & 0.00 \\
$t=0.1$ & 0.57945 & 0.23226 & 0.29235 \\
$t=0.2$ & 0.34554 & 0.22790 & 0.49526 \\
$t=0.3$ & 0.22200 & 0.17660 & 0.50051 \\
$t=0.4$ & 0.15708 & 0.13220 & 0.63007 \\
$t=0.5$ & 0.12364 & 0.10420 & 0.65919 \\
$t=0.6$ & 0.10530 & 0.06465 & 0.70740 \\
$t=0.7$ & 0.19586 & 0.04311 & 0.70311 \\
$t=0.8$ & 0.10088 & 0.02750 & 0.75970 \\
$t=0.9$ & 0.08861 & 0.01640 & 0.74071 \\
\hline
\end{tabular}

TABLE 2: Numerical solution of the proposed model using $R K 4$ at classical order $\alpha=1$.

\begin{tabular}{lcrr}
\hline Time (week) & $S(t)$ & $I(t)$ & $R(t)$ \\
\hline$t=0$ & 1.00000 & 0.20000 & 0.00 \\
$t=0.1$ & 0.57735 & 0.24227 & 0.30235 \\
$t=0.2$ & 0.35667 & 0.22792 & 0.50527 \\
$t=0.3$ & 0.23300 & 0.18672 & 0.64052 \\
$t=0.4$ & 0.16808 & 0.14221 & 0.73009 \\
$t=0.5$ & 0.13374 & 0.10423 & 0.78910 \\
$t=0.6$ & 0.11546 & 0.07485 & 0.82784 \\
$t=0.7$ & 0.10581 & 0.05317 & 0.85318 \\
$t=0.8$ & 0.10088 & 0.03756 & 0.86971 \\
$t=0.9$ & 0.09853 & 0.02646 & 0.88047 \\
\hline
\end{tabular}

In addition, we give a comparison of $R K 4$ and LADM in Tables 1 and 2 for $\alpha=1$, which shows that both the methods agree for short interval of time. The proposed method is better than RK4 method as it needs no extra predefined parameter which controls the method.

\section{Convergence Analysis}

Solution (16) is in the form of series, which converges uniformly to the exact solution. To check the convergence of series (16), we use techniques (see [14]). For sufficient conditions of convergence of this method, we give the following theorem by using [14].

Theorem 3 (see [13]). Let $\mathscr{X}$ be the Banach space and $\phi: \mathscr{X} \rightarrow$ $\mathscr{X}$ be a contractive nonlinear operator such that, for all $x, x^{\prime} \in$ $\mathscr{X},\left\|\phi(x)-\phi\left(x^{\prime}\right)\right\| \leq k\left\|x-x^{\prime}\right\|, 0<k<1$.

By using Banach contraction principles $\phi$ has a unique point $x$ such that $\phi x=x$, where $x=(S, I, R)$. The series given in (16) can be written by applying Adomian Decomposition Method as follows:

$$
x_{n}=\phi x_{n-1}, \quad x_{n-1}=\sum_{i=1}^{n-1} x_{i}, n=1,2,3, \ldots
$$

and assume that $x_{0}=x_{0} \in B_{r}(x)$, where $B_{r}(x)=x^{\prime} \in \mathscr{X}$ : $\left\|x^{\prime}-x\right\|<r$; then, we have

$$
\begin{aligned}
& \text { (1) } x_{n} \in B_{r}(x) \text {; } \\
& \text { (2) } \lim _{n \rightarrow \infty} x_{n}=x \text {. }
\end{aligned}
$$

Theorem 4 (see [15]). Let $f$ be an operator from a Hilbert space $H$ into $H$ and $y$ be the exact solution of (5), where $y=$ $(S(t), I(t), R(t)) . \sum_{i=0}^{\infty} y_{i}$, which is obtained by (9), converges to $y$ when $\exists, 0 \leq \alpha<1$ and $\left\|y_{k+1}\right\| \leq \alpha\left\|y_{k}\right\|, \forall k \in N U\{0\}$.

\section{Conclusion}

In this paper, we have considered a fractional order childhood disease model. The concern model is investigated for numerical solution by using LADM. The LADM is effective tool to solve nonlinear models and is widely used in engineering and applied mathematics. Also we have provided convergence results for the aforesaid method. It is clear that this method provides good results. One can observe that the method provides excellent numerical solutions for nonlinear fractional order models as compared to other methods like homotopy analysis, homotopy perturbation method, and RK4. Since these methods involve an extra parameter $h$ on which the solutions depend, therefore our proposed method needs no 
parameter and is easy to understand as well as to implement. For initial value problems, our proposed method is better than the PDQM and cubic-spline DQM methods. As PDQM, cubic-spline DQM methods are based on discretization technique which needs extra memory and time as compared to LADM. The method includes a pseudospectral method referred to as the quadrature discretization method (PQDM) and cubic-spline DQM, which are based on nonstandard polynomial basis sets. The aforesaid method is slowly converging as compared to LADM [16].

\section{Conflicts of Interest}

The authors declared that no conflicts of interest exist regarding this paper.

\section{Authors' Contributions}

All authors have equal contributions and they read and approved the final version of the paper. In the review process Dr. Shakoor Muhammad has provided valuable comments and suggestions and finally he checked and approved the final version. The review process without him was not possible.

\section{References}

[1] A. A. M. Arafa, S. Z. Rida, and M. Khalil, "Solutions of fractional order model of childhood diseases with constant vaccination strategy," Mathematical Science Letter, vol. 1, no. 1, pp. 17-23, 2012.

[2] H. Singh, J. Dhar, H. S. Bhatti, and S. Chandok, "An epidemic model of childhood disease dynamics with maturation delay and latent period of infection," Modeling Earth Systems and Environment, vol. 2, no. 79, 2016.

[3] R. H. Henderson, "The expanded programme on immunization of the world health organization," Reviews of Infectious Diseases, vol. 6, no. 2, pp. 475-479, 1984.

[4] O. D. Makinde, "Adomian decomposition approach to a SIR epidemic model with constant vaccination strategy," Applied Mathematics and Computation, vol. 184, no. 2, pp. 842-848, 2007.

[5] C. F. Li, X. N. Luo, Y. Zhou, and J. J, "Existence of, positive solution of the boundary value problem for nonlinear fractinal differentialn equations," Computer and Mathematics with Applications, vol. 59, pp. 1363-1375, 2010.

[6] F. Haq, K. Shah, G.-U. Rahman, and M. Shahzad, "Numerical analysis of fractional order model of HIV-1 infection of CD4+ T-cells," Computational Methods for Differential Equations, vol. 5, no. 1, pp. 1-11, 2017.

[7] A. Babakhani and V. Daftardar-Gejji, "Existence of positive solutions of nonlinear fractional differential equations," Journal of Mathematical Analysis and Applications, vol. 278, no. 2, pp. 434-442, 2003.

[8] M. Benchohra, S. Hamani, and S. K. Ntouyas, "Boundary value problems for differential equations with fractional order and nonlocal conditions," Nonlinear Analysis: Theory, Methods \& Applications, vol. 71, no. 7-8, pp. 2391-2396, 2009.

[9] D. Kumar and J. Singh, "Sushela, Application of homotopy analysis transform method to fractional biological population model," Romanian Reports Physics, vol. 65, no. 1, pp. 63-75, 2013.
[10] F. Haq, K. Shah, G. Rahman, and M. Shahzad, "Existence and uniqueness of positive solution to boundary value problem of fractional differential equations," Sindh Univ. Res. Jour, vol. 48, no. 2, pp. 451-456, 2016.

[11] A. Yildirim and Y. Cherruault, "Analytical approximate solution of a SIR epidemic model with constant vaccination strategy by homotopy perturbation method," Kybernetes, vol. 38, no. 9, pp. 1566-1575, 2009.

[12] Y. Lia, F. Haq, K. Shah, M. Shahzad, and G. Rahman, "Numerical analysis of fractional order Pine wilt disease model with bilinear incident rate," Journal of Mathematics and Computer Science, vol. 17, pp. 420-428, 2017.

[13] K. Shah, H. Khalil, and R. A. Khan, "Analytical solutions of fractional order diffusion equations by natural transform method," Iranian Journal of Science \& Technology, vol. 2016, pp. 1-13, 2016.

[14] K. Shah, H. Khalil, and R. A. Khan, "Analytical solutions of fractional order diffusion equations by natural transform method," Iranian Journal of Science \& Technology, vol. 2016, 14 pages, 2016.

[15] M. M. Hosseini and H. Nasabzadeh, "On the convergence of Adomian decomposition method," Applied Mathematics and Computation, vol. 182, no. 1, pp. 536-543, 2006.

[16] K. Leung, B. D. Shizgal, and H. Chen, "The quadrature discretization method (QDM) in comparison with other numerical methods of solution of the Fokker-Planck equation for electron thermalization," Journal of Mathematical Chemistry, vol. 24, no. 4, pp. 291-319, 1998. 


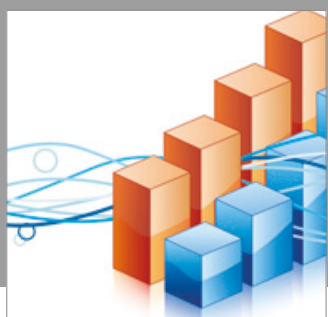

Advances in

Operations Research

vatersals

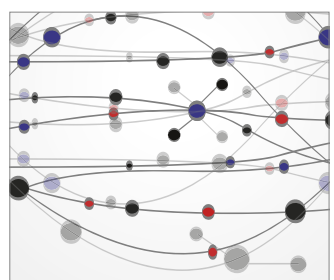

\section{The Scientific} World Journal
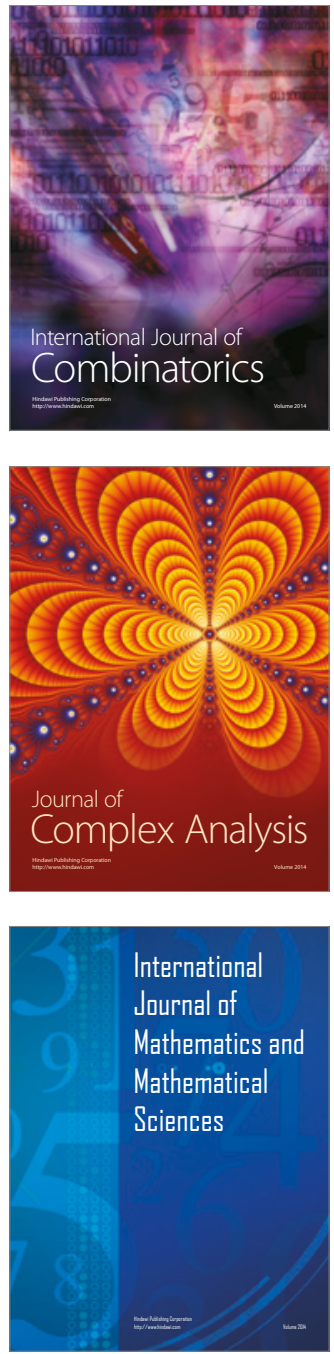
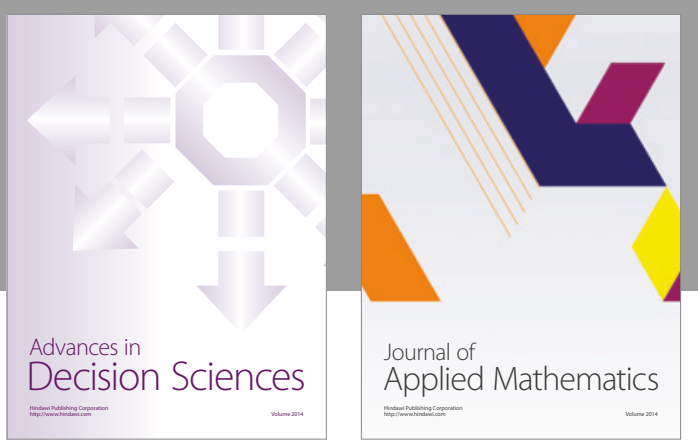

Algebra

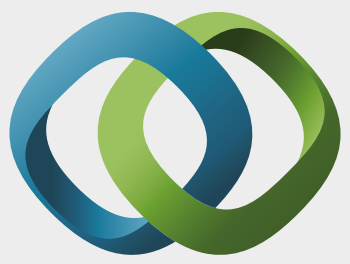

\section{Hindawi}

Submit your manuscripts at

https://www.hindawi.com
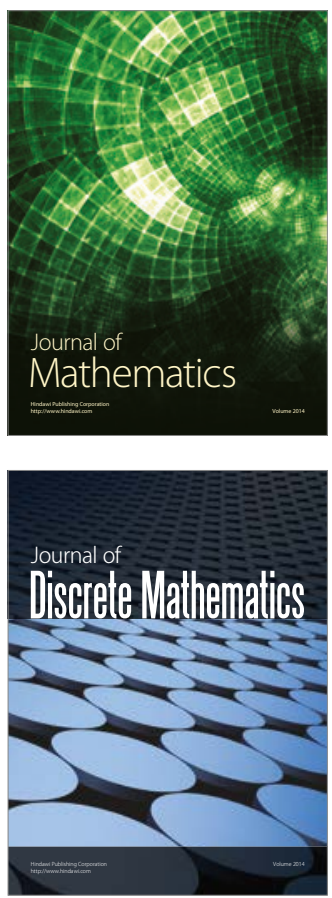

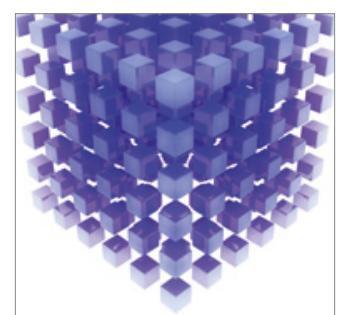

Mathematical Problems in Engineering
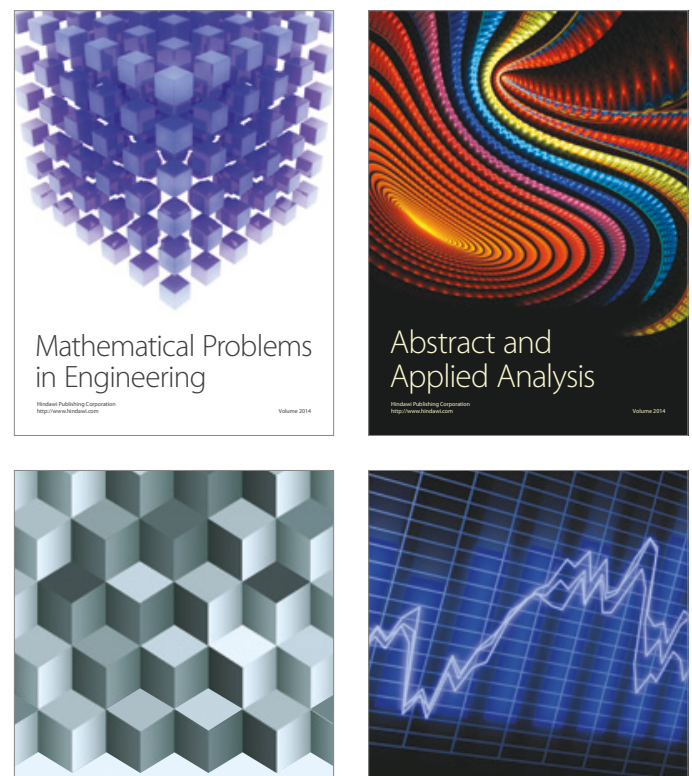

Journal of

Function Spaces

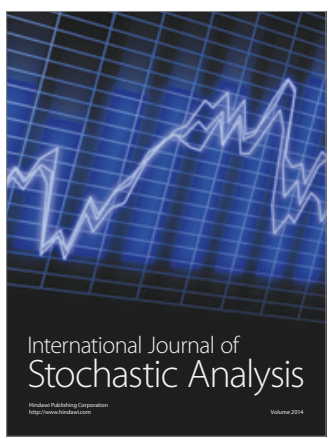

Probability and Statistics
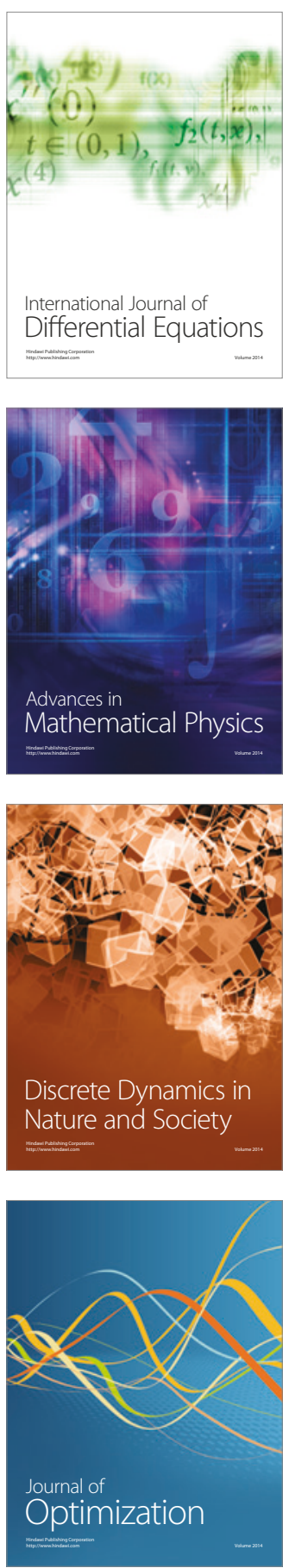